\title{
Stellar Populations and Local Group Membership of the Dwarf Galaxy DDO 210
}

\author{
Myung Gyoon Lee \\ Department of Astronomy, Seoul National University, Korea
}

\begin{abstract}
Deep $B V I$ CCD photometry of dwarf galaxy DD0 210 obtained in this study shows that the resolved stellar population in this galaxy consists of a dominant old red giant population and some young stars. We have estimated the distance to this galaxy using the $I$-band magnitude of the tip of the red giant branch, obtaining a value of $1030 \pm 50$ kpc. This result combined with the velocity of DDO 210 shows that DDO 210 is a member of the Local Group.
\end{abstract}

\section{Introduction}

DDO 210 is a dwarf irregular galaxy discovered by van den Bergh in 1959. The distance to this galaxy has been known to be very uncertain, with literature values ranging from $1 \mathrm{Mpc}$ to $4 \mathrm{Mpc}$ (Fisher \& Tully 1975, Greggio et al. 1993). Correspondingly it has been sometimes considered to be a member of the Local Group and sometimes not (van den Bergh 1994). We present a study of stellar populations and the Local Group membership of DDO 210 using deep BVI photometry.

\section{Observations and Data Reduction}

$B V I$ CCD images of DDO 210 were obtained on the photometric night of August 22, 1992 at the Palomar $1.5 \mathrm{~m}$ telescope and on the clear night of October 8, 1994 at the University of Hawaii $2.2 \mathrm{~m}$ telescope.

Instrumental magnitude of the stars were derived using DoPHOT (Schechter et al. 1993), and they were transformed to the standard system using the Landolt standard stars observed on the same night of August 22, 1992.

A color image of DDO 210 created by combining $B, V$ and $I$ images shows that DDO 210 is well resolved into stars and has an elongated structure with a size $4^{\prime} .8 \times 2^{\prime} .1$. Most of the bright stars seen in the main body $\left(2^{\prime} .3 \times 0^{\prime} .9\right)$ of DDO 210 are blue, showing that they are young stars, and there are many faint yellow stars extending farther from the main body, which are mostly red giants.

\section{Color-Magnitude Diagrams}

The color-magnitude diagrams of about 900 measured stars in the central region of DDO 210 are displayed in Fig. 1. It shows (a) there is a well-defined red giant 


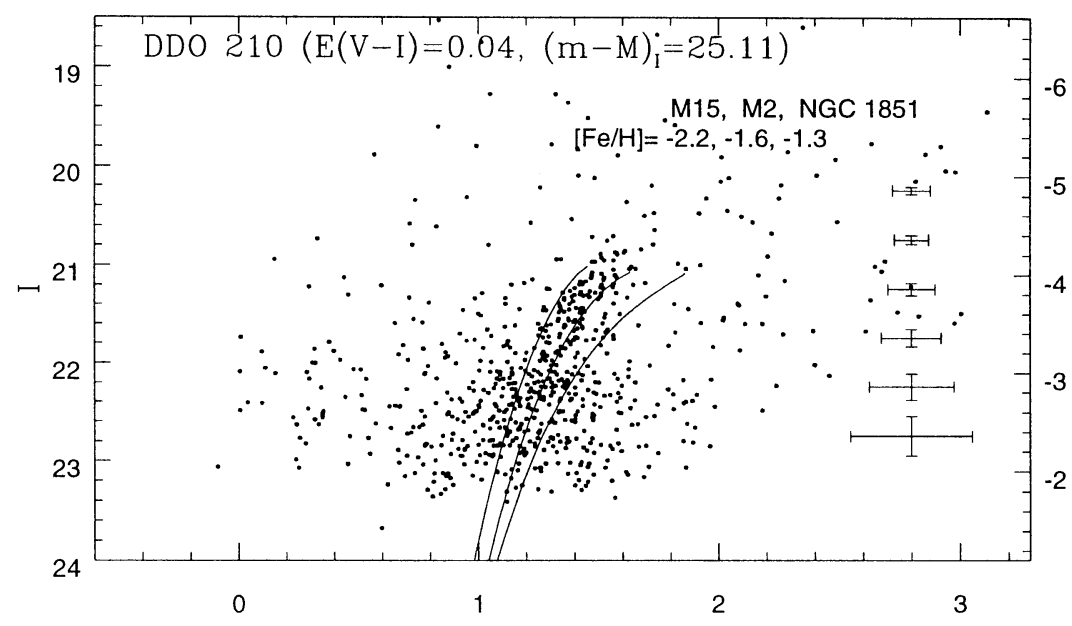

$(\mathrm{V}-\mathrm{I})$

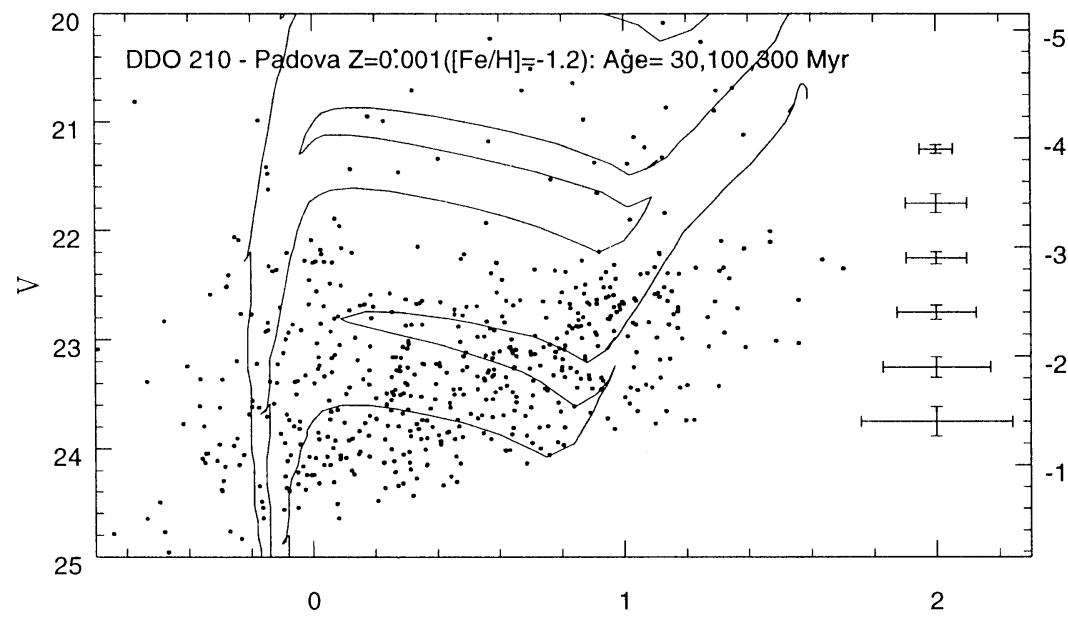

(B-V)

Figure 1. (Top) $I-(V-I)$ diagram of the measured stars in DDO 210. The solid lines represent, from left to right, the loci of the red giant branch of the Galactic globular clusters: M15, M2 and NGC 1851, shifted according to the distance determined in this study. The metallicities of these clusters are $[\mathrm{Fe} / \mathrm{H}]=-2.2,-1.6$ and -1.3 dex, respectively. The mean photometric errors are represented by error bars at the right side. The right axis represents the absolute magnitude, $M_{I}$. (Bottom) $V-(B-V)$ diagram of the measured stars in DDO 210. The solid lines represent the Padova theoretical isochrones for $[\mathrm{Fe} / \mathrm{H}]=-1.2$ and ages 30,100 , and $300 \mathrm{Myr}$, shifted according to the distance determined in this study. The right axis represents the absolute magnitude, $M_{V}$. 

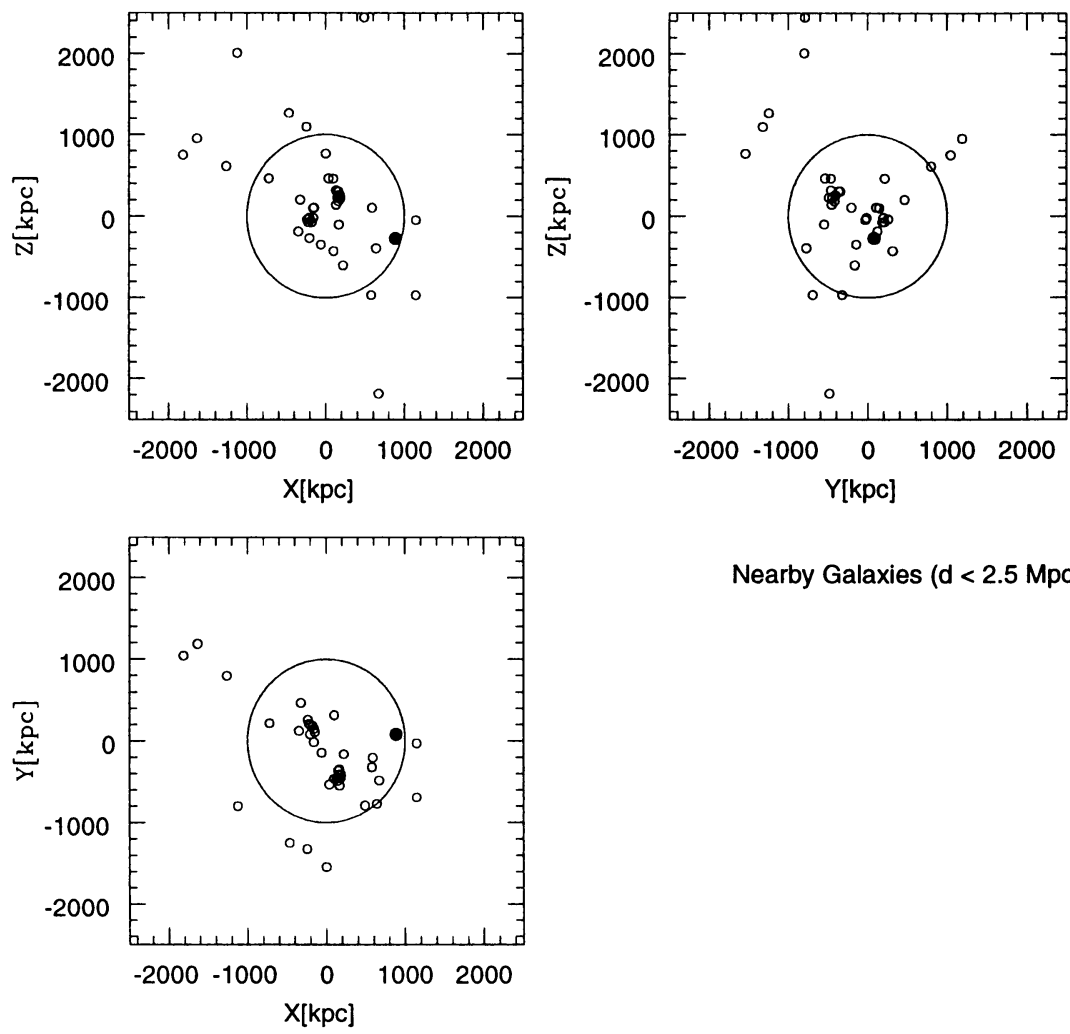

Nearby Galaxies (d $<2.5 \mathrm{Mpc})$

Figure 2. Projections of nearby galaxies (open circles) closer than $2.5 \mathrm{Mpc}$ on the XYZ planes. The center of the planes is at the center of the Local Group and the radius of the large circle is $1 \mathrm{Mpc}$. DDO 210 is marked by a filled circle. 
branch (RGB) reaching up to $I \approx 21 \mathrm{mag}$, indicating a presence of an old stellar population, and (b) there is a blue plume extending up to $V \approx 22$ mag, with a few stars above, which consists of mostly young main sequence and supergiant stars.

\section{Distance and Local Group Membership}

We have estimated the distance to DDO 210 using the $I$ magnitude of the tip of the RGB (TRGB), an accurate distance indicator, as described in Lee et al. (1993) and Lee (1996). The $I$ magnitude of the TRGB is estimated to be $I=21.15 \pm 0.10 \mathrm{mag}$ from the luminosity function of red giants. Using this value and adopting the foreground reddening of $E(B-V)=0.03$, we have derived a value for the distance: $(m-M)_{0}=25.05 \pm 0.11$ and $d=1030 \pm 50 \mathrm{kpc}$.

We have also estimated the mean metallicity of the RGB, obtaining a value of $[\mathrm{Fe} / \mathrm{H}] \approx-2.0 \mathrm{dex}$, from the mean color of the RGB. The RGB of DDO 210 is compared with the RGBs of Galactic globular clusters M15, M2 and NGC $1851([\mathrm{Fe} / \mathrm{H}]=-2.2,-1.6$ and $-1.3 \mathrm{dex}$, respectively) in Fig. 1a. This shows that the RGB of DDO 210 is located between those of M15 and M2. The RGB of DDO 210 is quite metal-poor, which is typical for the RGBs in dwarf galaxies.

The absolute magnitude of DDO 210 is derived to be $M_{B}=-9.8 \mathrm{mag}$, which is close to the faint end of the Local Group galaxies. The low luminosity, small size and rather elliptical structure of DDO 210 indicate that this galaxy is intermediate between the dwarf irregular and dwarf spheroidal types, as are LGS-3 and Phoenix (Lee 1995a).

From the distance measured above, we have derived the position of DDO 210 with respect to the center of the Local group. The distance of DDO 210 from the center of the Local Group is derived to be $930 \mathrm{kpc}$. Figure 2 shows the projection of DDO 210 and other nearby galaxies closer than $2.5 \mathrm{Mpc}$ in the XYZ planes, using the data compiled in Lee (1995b). DDO 210 does not belong to either the M31 group or our Galaxy group, but is an isolated galaxy. Considering this distance and the heliocentric velocity of DDO $210\left(-136 \mathrm{~km} \mathrm{~s}^{-1}\right)$, we conclude that DDO 210 is a member of the Local Group.

\section{References}

Fisher, J.R., Tully, R.B. 1975, A\&A, 44, 151

Greggio, L., Marconi, G., Tosi, M., Focardi, P. 1993, AJ, 105, 894

Lee, M.G., Freedman, W.L., Madore, B.F. 1993, ApJ, 417, 553

Lee, M.G. 1995a, AJ, 110, 1129

Lee, M.G. 1995b, Jour. Korean Astro. Soc., 28, 169

Lee, M.G. 1996, Jour. Korean Astro. Soc., 29, S67

Schechter, P., Mateo, M., Saha, A. 1993, PASP, 105, 1342

van den Bergh, S. 1994, AJ, 107, 1328 